\title{
有理函数的给定周期的排斥周期点的 存在性*
}

$$
\text { 方明亮* }
$$

(华南农业大学应用数学系, 广州 510642)
常建明

(常熟理工学院数学系, 常熟 215500)

摘要 设 $R$ 为一次数 $d \geqslant 2$ 的有理函数, $k \geqslant 2$ 为一正整数, 则 $R$ 至少有一个 周期为 $k$ 的排斥周期点, 除非 $k=4$ 且 $d=2$, 或 $k=3$ 且 $d \leqslant 3$, 或 $k=2$ 且 $d \leqslant 8$. 例子表明所有的例外情形都会出现.

\section{关键词 有理函数 迭代 不动点 周期点 排斥周期点}

\section{1 引言与主要结果}

设 $R(z)$ 为一有理函数, $k$ 为一整数, 则 $R(z)$ 的 $k$ 次迭代, 记作 $R^{k}(z)$, 可归 纳地定义为 $R^{1}(z)=R(z)$ 和当 $k \geqslant 2$ 时 $R^{k}(z)=R^{k-1} \circ R(z)$.

设 $z_{0} \in \overline{\mathbb{C}}$. 如果存在正整数 $p \in \mathbb{N}$, 使得 $R^{p}\left(z_{0}\right)=z_{0}$, 则称满足该式最小的 正整数 $p$ 为 $R(z)$ 的一个周期, $z_{0}$ 称为 $R(z)$ 的一个周期为 $p$ 的周期点, 并且称 相应的轨道 $\left\{z_{0}, R\left(z_{0}\right), \cdots, R^{p-1}\left(z_{0}\right)\right\}$ 为 $R(z)$ 的一个周期为 $p$ 的周期轨道. 特别 地, 周期为 1 的周期点称为不动点.

设 $z_{0} \in \overline{\mathbb{C}}$ 为 $R(z)$ 的一个周期为 $p$ 的周期点. 当 $z_{0} \neq \infty$ 时, 定义 $z_{0}$ (和相应 轨道) 的乘子为 $\lambda=\left(R^{p}\right)^{\prime}\left(z_{0}\right)$; 而当 $z_{0}=\infty$ 时, 定义乘子为 $\lambda=\lim _{z \rightarrow \infty} \frac{1}{\left(R^{p}\right)^{\prime}(z)}$ (参见文献 [1] p.23). 进一步地, 按照 $|\lambda|<1,|\lambda|=1$ 或 $|\lambda|>1$, 称周期点 $z_{0}$ (和相 应轨道) 是吸引的, 中性的或排斥的. 在中性的情形, 则按照是否存在一个正整 数 $m$, 使得 $(\lambda)^{m}=1$, 周期点 $z_{0}$ (和相应轨道) 称为有理中性或无理中性的 (参见文 献 [2] p.27 和 p.54).

收稿日期: 2005-10-05; 接受日期: 2006-04-06

* 国家自然科学基金 (批准号: 10471065), 教育部留学回国人员启动基金及江苏省教育厅科研基金 (04KJD110001) 资助项目

**E-mail: hnmlfang@hotmail.com 
周期点, 特别是排斥周期点在复解析动力系统中起着非常重要的作用. 例 如, 有理函数的 Julia 集是排斥周期点集合的闭包.

Baker ${ }^{[3]}$ 最先研究了具有指定周期的周期点的存在性问题, 他证明了

定理 $\mathbf{A}$ 设 $P(z)$ 是次数为 $d \geqslant 2$ 的多项式, $k \geqslant 2$ 为一正整数, 则 $P(z)$ 至 少有一个周期为 $k$ 的周期点, 除非 $(k, d)=(2,2)$ 并且 $P(z)$ 相似于 $z^{2}-3 / 4$.

这里称两多项式 $P(z)$ 和 $Q(z)$ 相似, 如果存在常数 $a(\neq 0)$ 和 $b$, 使得 $Q(a z+$ $b)=a P(z)+b$.

定理 $\mathbf{B}$ 设 $R(z)$ 是次数为 $d \geqslant 2$ 的有理函数, $k \geqslant 2$ 为一正整数, 则 $R(z)$ 至少有一个周期为 $k$ 的周期点, 除非 $(k, d) \in\{(2,2),(2,3),(2,4),(3,2)\}$. 所有的 例外情形都会出现.

Bergweiler ${ }^{[4,5]}$ 对多项式研究了具有指定周期的排斥周期点的存在性问题, 他证明了

定理 $\mathbf{C}$ 设 $P(z)$ 是次数为 $d \geqslant 2$ 的多项式, $k \geqslant 2$ 为一正整数, 则 $P(z)$ 至 少有一个周期为 $k$ 的排斥周期点, 除非 $(k, d) \in\{(2,2),(2,3),(2,4),(3,2)\}$. 所有 的例外情形都会出现.

定理 D 设 $P(z)$ 是次数为 $d \geqslant 2$ 的多项式, $k \geqslant 2$ 为一正整数. 记 $N_{r p}(k)$ 为 $P$ 的周期为 $k$ 的排斥周期点个数, $N_{p}(k)$ 为 $P$ 的周期为 $k$ 的周期点个数, 则

$$
N_{r p}(k) \geqslant d^{k}-\sum_{j \mid k, j<k} d^{j}-2 k(d-1), \quad N_{p}(k) \geqslant d^{k}-\sum_{j \mid k, j<k} d^{j}-k(d-1) .
$$

自然地, 我们需要研究有理函数的具有指定周期的排斥周期点的存在性问 题. 本文研究这个问题, 证明了

定理 1 设 $R(z)$ 是次数为 $d \geqslant 2$ 的有理函数, $k \geqslant 2$ 为一正整数, 则 $R(z)$ 至少 有一个周期为 $k$ 的排斥周期点, 除非 $(k, d) \in\{(2,2),(2,3),(2,4),(2,5),(2,6),(2,7)$, $(2,8),(3,2),(3,3),(4,2)\}$. 所有的例外情形都会出现.

定理 1 的证明实际上还给出了如下关于具有指定周期的 (排斥) 周期点个数 的估计.

定理 2 设 $R(z)$ 是次数为 $d \geqslant 2$ 的有理函数, $k \geqslant 2$ 为一正整数. 记 $N_{r p}(k)$ 为 $R$ 的周期为 $k$ 的排斥周期点个数, $N_{p}(k)$ 为 $R$ 的周期为 $k$ 的周期点个数, 则 有

$$
N_{r p}(k) \geqslant d^{k}-\sum_{j \mid k, j<k} d^{j}-4 k(d-1), \quad N_{p}(k) \geqslant d^{k}-\sum_{j \mid k, j<k} d^{j}-2 k(d-1) .
$$

\section{2 定理 1 例外情形的几个例子}

这一节中, 我们用例子来说明定理 1 所说的例外情形都会出现. 由 Baker 的 定理 B 及他的例子, 只要考虑 $(k, d) \in\{(4,2),(3,3),(2,5),(2,6),(2,7),(2,8)\}$ 的情 形. 下面的例 2 也表明定理 2 中的等号能够成立. 例子的计算较复杂, 在附录中 
我们选择计算最复杂的例 6 , 就该例的计算过程作了一个说明.

例 1 设

$$
R(z)=z+\frac{(-2+\mathrm{i} \sqrt{2})\left(3 z^{2}+2-2 \mathrm{i} \sqrt{2}\right)}{6 z},
$$

则 $R$ 没有周期为 4 的排斥周期点.

例 2 设

$$
R(z)=z-\frac{12+8 \mathrm{i}}{13} \cdot \frac{z^{3}-(13+234 \mathrm{i}) z}{z^{2}+145+10 \mathrm{i}}
$$

则 $R$ 没有周期为 3 的排斥周期点. 注意此例也表明定理 2 中的等号能够成立.

例 3 设

$$
R(z)=z+\frac{8 z\left(z^{2}-1+\mathrm{i}\right)}{z^{4}-(4-4 \mathrm{i}) z^{2}-4 \mathrm{i}},
$$

则 $R$ 没有周期为 2 的排斥周期点.

例 4 设

$$
\begin{aligned}
R(z) & =z+\frac{(c-1) z^{6}+\left(378 c^{5}+3591 c^{4}+1116 c^{3}+1141 c^{2}-164 c-77\right) z}{z^{5}+45} \\
& =z+(c-1) \frac{z^{6}+\frac{378 c^{5}+3591 c^{4}+1116 c^{3}+1141 c^{2}-164 c-77}{c-1} z}{z^{5}+45},
\end{aligned}
$$

这里 $c$ 是常数, 满足 $189 c^{6}+189 c^{5}+180 c^{4}+80 c^{3}+30 c^{2}+6 c+1=0$, 则 $R$ 没有 周期为 2 的排斥周期点.

例 5 设

$$
R(z)=z+\frac{(1-c) z^{7}-(7+5 c) z}{c z^{6}+3+2 c}=z+\left(\frac{1}{c}-1\right) \frac{z^{7}-\frac{7+5 c}{1-c} z}{z^{6}+\frac{3+2 c}{c}},
$$

这里 $c$ 是常数，满足 $c^{4}+2 c^{3}+3 c^{2}+8 c+7=0$, 则 $R$ 没有周期为 2 的排斥周期 点.

例 6 设

$$
R(z)=z+\frac{(c-1) z^{8}+a z}{z^{7}+2882600}=z+(c-1) \frac{z^{8}+\frac{a}{c-1} z}{z^{7}+2882600},
$$

这里 $c$ 和 $a$ 是常数, 满足

$$
1536 c^{8}+1536 c^{7}+1120 c^{6}+840 c^{5}+721 c^{4}+308 c^{3}+126 c^{2}+60 c+25=0
$$

和

$$
\begin{aligned}
a= & -86017536 c^{7}+156417024 c^{6}-67928160 c^{5}-56966440 c^{4} \\
& -17020121 c^{3}+3791327 c^{2}-24046371 c-5852675,
\end{aligned}
$$

则 $R$ 没有周期为 2 的排斥周期点. 


\section{3 引理}

引理 $\mathbf{1}^{[6,7]}$ (参见文献 [8] 引理 25]) 任一次数不小于 2 的有理函数至少有一 个排斥不动点或有一个乘子为 1 的不动点.

具有引理 1 所述性质的不动点称为弱排斥不动点 (参见文献 [8-10]).

引理 $2^{[11]}$ 设 $R(z)$ 是次数为 $d \geqslant 2$ 的有理函数, 则 $R(z)$ 至多有 $2 d-2$ 个非 排斥周期轨道.

设 $\left\{z_{0}, R\left(z_{0}\right), \cdots, R^{p-1}\left(z_{0}\right)\right\} \subset \mathbb{C}$ 是 $R$ 的一个周期为 $p$ 的有理中性轨道，则 存在一个最小的整数 $m \geqslant 1$, 使得 $\left[\left(R^{p}\right)^{\prime}\left(z_{0}\right)\right]^{m}=1$. 于是存在唯一的常数 $c \neq 0$ 和 唯一的正整数 $k$, 使得在 $z_{0}$ 的邻域内

$$
R^{p m}(z)=z+c\left(z-z_{0}\right)^{k m+1}[1+o(1)]
$$

(参见文献 [2] p. 41 和文献 [5] p. 8). 进一步地，对任意正整数 $n$, 有

$$
R^{n p m}(z)=z+n c\left(z-z_{0}\right)^{k m+1}[1+o(1)] .
$$

根据 Leau-Fatou 花瓣定理, 对每个 $0 \leqslant j \leqslant p-1, R$ 的 Fatou 集有 $k m$ 个分支 $U_{j, i}(1 \leqslant i \leqslant k m)$, 使得 $R^{j}\left(z_{0}\right)$ 在 $U_{j, i}$ 的边界上, 并且在 $U_{j, i}$ 内, $R^{n p}(z) \rightarrow R^{j}\left(z_{0}\right)$, $(n \rightarrow \infty)$. 这些 $U_{j, i}$ 叫做 Leau 域或吸引花瓣, 它们可分成 $k$ 组, 每组 $p m$ 个, 并且每一组都是 $R$ 不变的. 即每一组可以写成形式 $\left\{R^{j}(U), 0 \leqslant j \leqslant p m-1\right\}$ $\left(R^{p m}(U)=U\right.$ ), 称为一组吸引花瓣循环 (参见文献 [5] p. 8, 文献 [2] p. 60 和文献 [1] p. 41-43).

引理 3( 文献 [2] 定理 III.2.3) $\quad R(z)$ 的任一组吸引花瓣循环至少包含一个临 界点, 其中 $R(z)$ 是次数为 $d \geqslant 2$ 的有理函数.

注意一个熟知的事实, $R(z)$ 的临界点至多 $2 d-2$ 个, 这里 $d$ 是 $R(z)$ 的次数 (参见文献 [2] p.54).

引理 4 设 $R$ 是一个次数不小于 2 的有理函数, $\infty$ 为它的弱排斥不动点, 则 $R$ 可以写成

$$
R(z)=z+c \frac{Q(z)}{P(z)},
$$

这里 $c \neq 0$ 为常数, $P(z)$ 和 $Q(z)$ 为两个互质的首一多项式, 次数分别为 $p$ 和 $q$ 满足 $q \leqslant p+1$, 并且当 $q=p+1$ 时, $0<|c+1|<1$.

进而对任意 $n \in \mathbb{N}, R^{n}$ 具有形式

$$
R^{n}(z)=z+c_{n} \frac{Q_{n}(z)}{P_{n}(z)},
$$

这里 $c_{n} \neq 0$ 为常数, $P_{n}(z)$ 和 $Q_{n}(z)$ 为两个互质的首一多项式, 并且

$$
\operatorname{deg}\left(Q_{n}\right)=q+(p+1)^{n}-(p+1), \quad \operatorname{deg}\left(P_{n}\right)=(p+1)^{n}-1 .
$$

证明 先证引理的前半部分. 将 $R$ 写成

$$
R(z)=c^{\prime} \frac{U(z)}{P(z)}
$$


这里 $c^{\prime} \neq 0$ 为常数, $P(z)$ 和 $U(z)$ 为两个互质的首一多项式, 次数分别为 $p$ 和 $u$, 于是 $\operatorname{deg}(R)=\max \{p, u\} \geqslant 2$.

由于 $R(\infty)=\infty$, 由 (6) 式知 $u \geqslant p+1$. 于是在 $\infty$ 处,

$$
R(z)=c^{\prime} z^{u-p}[1+o(1)] .
$$

由 (7) 式知, 如果 $u-p \geqslant 2$, 则 $\infty$ 的乘子为 0 , 因而是 $R$ 的吸引不动点, 与假设 矛盾, 从而 $u-p \leqslant 1$, 于是 $u=p+1$. 再由 (7) 式知 $\infty$ 的乘子为 $1 / c^{\prime}$. 记 $\widetilde{Q}(z)=$ $c^{\prime} U(z)-z P(z)$, 则 $\widetilde{Q}$ 是不恒为零的多项式, 与 $P$ 互质并且 $q=\operatorname{deg}(\widetilde{Q}) \leqslant p+1$, 此时

$$
R(z)=z+\frac{\widetilde{Q}(z)}{P(z)} .
$$

由条件, $\infty$ 的乘子 $1 / c^{\prime}$ 满足 $1 / c^{\prime}=1$ 或者 $\left|1 / c^{\prime}\right|>1$, 即 $c^{\prime}=1$ 或者 $0<\left|c^{\prime}\right|<1$. 若前者成立, 则 $q=\operatorname{deg}(\widetilde{Q}) \leqslant p$. 若后者成立, 则 $q=\operatorname{deg}(\widetilde{Q})=p+1$. 设 $\widetilde{Q}$ 的首项 系数为 $c$, 并记 $Q(z)=\widetilde{Q}(z) / c$. 注意在后一情形, $c=c^{\prime}-1$, 从而 $0<|c+1|<1$. 于是由 (8) 式知, $R$ 可写成形式 (3).

现在用归纳法来证明后半部分. 设

$$
\begin{aligned}
& P(z)=z^{p}+a_{1} z^{p-1}+\cdots+a_{p}, \\
& Q(z)=z^{q}+b_{1} z^{q-1}+\cdots+b_{q},
\end{aligned}
$$

这里 $a_{j}(1 \leqslant j \leqslant p)$ 和 $b_{j}(1 \leqslant j \leqslant q)$ 均为常数.

情形 $1 \quad p \geqslant q$. 显然, 对 $n=1$, 结论成立并且 $c_{1}=c$. 现设结论对 $n$ 成立并 且 $c_{n}=n c$. 下面证明结论对 $n+1$ 也成立并且 $c_{n+1}=(n+1) c$.

由归纳假设, 有

$$
\begin{aligned}
R^{n+1}(z) & =R\left(R^{n}(z)\right) \\
& =R^{n}(z)+c \frac{Q\left(R^{n}(z)\right)}{P\left(R^{n}(z)\right)} \\
& =z+n c \frac{Q_{n}(z)}{P_{n}(z)}+c \frac{Q\left(z+n c \frac{Q_{n}(z)}{P_{n}(z)}\right)}{P\left(z+n c \frac{Q_{n}(z)}{P_{n}(z)}\right)} \\
& =z+n c \frac{Q_{n}(z)}{P_{n}(z)}+c \frac{L(z)}{H(z)}\left(P_{n}(z)\right)^{p-q},
\end{aligned}
$$

这里

$$
\begin{aligned}
H(z) & =\left[P_{n}(z)\right]^{p} P\left(z+n c \frac{Q_{n}(z)}{P_{n}(z)}\right) \\
& =\left[z P_{n}(z)+n c Q_{n}(z)\right]^{p}+a_{1} P_{n}\left[z P_{n}(z)+n c Q_{n}(z)\right]^{p-1}+\cdots+a_{p}\left[P_{n}(z)\right]^{p} \\
& =z^{p(p+1)^{n}}+\cdots+h_{1} z+h_{0} \\
L(z) & =\left[P_{n}(z)\right]^{q} Q\left(z+n c \frac{Q_{n}(z)}{P_{n}(z)}\right)
\end{aligned}
$$




$$
\begin{aligned}
& =\left[z P_{n}(z)+n c Q_{n}(z)\right]^{q}+b_{1} P_{n}\left[z P_{n}(z)+n c Q_{n}(z)\right]^{q-1}+\cdots+b_{q}\left[P_{n}(z)\right]^{q} \\
& =z^{q(p+1)^{n}}+\cdots+l_{1} z+l_{0} .
\end{aligned}
$$

记

$$
\begin{aligned}
M(z) & =P_{n}(z) \cdot H(z), \\
N(z) & =n Q_{n}(z) \cdot H(z)+L(z) \cdot\left[P_{n}(z)\right]^{p-q+1} .
\end{aligned}
$$

我们断言 $M(z)$ 和 $N(z)$ 是互质的多项式. 若不然, 则存在 $z_{0} \in \mathbb{C}$, 使得 $M\left(z_{0}\right)=$ $N\left(z_{0}\right)=0$. 于是由 (18) 和 (19) 式及 $P_{n}$ 和 $Q_{n}$ 无公共零点知 $H\left(z_{0}\right)=0$. 如果 $P_{n}\left(z_{0}\right)=0$, 则由 (13) 式有 $Q_{n}\left(z_{0}\right)=0$. 这与 $P_{n}$ 和 $Q_{n}$ 无公共零点相矛盾. 如果 $P_{n}\left(z_{0}\right) \neq 0$, 则由 $N\left(z_{0}\right)=0$ 知 $L\left(z_{0}\right)=0$. 由此结合 (12) 和 (15) 式可推出 $P$ 和 $Q$ 有公共零点 $z_{0}+n c Q_{n}\left(z_{0}\right) / P_{n}\left(z_{0}\right)$. 这与 $P$ 和 $Q$ 互质矛盾.

于是 $M(z)$ 和 $N(z)$ 是互质的多项式, 并且由 (14) 和 (17)-(19) 式, 有

$$
\begin{aligned}
M(z) & =z^{(p+1)^{n+1}-1}+\cdots+m_{1} z+m_{0}, \\
N(z) & =(n+1) z^{q+(p+1)^{n+1}-(p+1)}+\cdots+n_{1} z+n_{0} .
\end{aligned}
$$

若记 $c_{n+1}=(n+1) c, P_{n+1}(z)=M(z)$ 和 $Q_{n+1}(z)=N(z) /(n+1)$, 则有

$$
R^{n+1}(z)=z+c_{n+1} \frac{Q_{n+1}(z)}{P_{n+1}(z)} .
$$

至此情形 1 证毕.

情形 $2 q=p+1$. 类似地可用数学归纳法加以证明, 并且 $c_{n}=(c+1)^{n}-1$.

\section{4 定理 1 与 2 的证明}

我们将先证明定理 2 , 再证明定理 1 .

定理 2 的证明 如果 $\infty$ 不是 $R$ 的弱排斥不动点, 则由引理 $1, R$ 有一个弱 排斥不动点 $z_{0} \in \mathbb{C}$. 记 $\phi(z)=z_{0}+1 /\left(z-z_{0}\right)$ 和 $\widetilde{R}=\phi^{-1} \circ R \circ \phi(z)$. 于是 $\infty$ 是 $\widetilde{R}$ 的弱排斥不动点. 显然, $R$ 和 $\widetilde{R}$ 具有相同的周期为 $k$ 的排斥周期轨道数, 因此 可以假设 $\infty$ 是 $R$ 的弱排斥不动点. 于是 $R$ 的所有周期不小于 2 的周期轨道均在 有限平面 $\mathbb{C}$ 内, 从而由引理 $4, R$ 可写成形式

$$
R(z)=z+c \frac{Q(z)}{P(z)},
$$

这里 $c$ 为非零常数, $P$ 和 $Q$ 是次数分别为 $p$ 和 $q$ 的互质的首一多项式，并且 $q \leqslant p+1$. 如果 $q=p+1$, 则还有 $0<|c+1|<1$, 于是 $d=\operatorname{deg}(R)=p+1$. 由引理 4 知, 对 $j \in \mathbb{N}$,

$$
R^{j}(z)=z+c_{j} \frac{Q_{j}(z)}{P_{j}(z)},
$$

这里 $c_{j} \neq 0$ 是常数, $P_{j}$ 和 $Q_{j}$ 是互质的首一多项式, 并且

$$
\operatorname{deg}\left(Q_{j}\right)=q+(p+1)^{j}-(p+1) .
$$


设 $l \in \mathbb{N}, z_{0} \in \mathbb{C}$ 是 $R^{l}$ 的一个不动点, 则有唯一的含有 $z_{0}$ 的周期轨道

$$
\left\{R^{0}\left(z_{0}\right)\left(=z_{0}\right), R^{1}\left(z_{0}\right), \cdots, R^{j-1}\left(z_{0}\right)\right\},
$$

它的周期为 $j$. 易见 $j$ 是 $l$ 的一个因数. 注意, 这条轨道在有限平面 $\mathbb{C}$ 内, 并且轨 道内的每一个点 $R^{s}\left(z_{0}\right)(0 \leqslant s \leqslant j-1)$ 都是 $R^{l}$ 的不动点, 而且由文献 [10] p.143 知, 它们作为方程 $R^{l}(z)-z=0$ 的解具有相同的重数.

现在设 $R$ 有 $n_{j} \geqslant 0$ 条周期为 $j$ 的周期轨道

$$
\Gamma_{j, i}=\left\{R^{0}\left(z_{j, i}\right), R^{1}\left(z_{j, i}\right), \cdots, R^{j-1}\left(z_{j, i}\right)\right\} \subset \mathbb{C}, \quad i=1,2, \cdots, n_{j},
$$

并且假设在这些轨道中, 有 $0 \leqslant m_{j} \leqslant n_{j}$ 条非排斥周期轨道 $\Gamma_{j, i}\left(1 \leqslant i \leqslant m_{j}\right)$, 于 是有

$R^{l}(z)=z+\frac{\prod_{j \mid l}\left\{\prod_{i=1}^{m_{j}}\left[\prod_{\zeta \in \Gamma_{j, i}}(z-\zeta)\right]^{\mu_{j, i}(l)+1} \prod_{i=m_{j}+1}^{n_{j}}\left[\prod_{\zeta \in \Gamma_{j, i}}(z-\zeta)\right]\right\}}{H(z)}$,

这里 $\mu_{j, i}(l)$ 是非负整数, $H(z)$ 是一多项式, 它没有零点落在 $\left\{\Gamma_{j, i}\right\}$ 中的任一轨 道内. 于是由 (21) 和 (23) 式,

$$
Q_{l}(z)=\prod_{j \mid l}\left\{\prod_{i=1}^{m_{j}}\left[\prod_{\zeta \in \Gamma_{j, i}}(z-\zeta)\right]^{\mu_{j, i}(l)+1} \prod_{i=m_{j}+1}^{n_{j}}\left[\prod_{\zeta \in \Gamma_{j, i}}(z-\zeta)\right]\right\} .
$$

因此由引理 4 和 (24) 式得

$$
\sum_{j \mid l} j\left(n_{j}+\sum_{i=1}^{m_{j}} \mu_{j, i}(l)\right)=q+(p+1)^{l}-(p+1) .
$$

特别地

$$
\ln _{l}(l) \leqslant q+(p+1)^{l}-(p+1) .
$$

于是有

$$
\sum_{j \mid k, j<k} j n_{j} \leqslant \sum_{j \mid k, j<k}\left[q+(p+1)^{j}-(p+1)\right] .
$$

从而由 (25) 和 (27) 式得

$$
\begin{aligned}
k n_{k} & =\sum_{j \mid k} j\left(n_{j}+\sum_{i=1}^{m_{j}} \mu_{j, i}(k)\right)-\sum_{j \mid k, j<k} j n_{j}-\sum_{j \mid k} \sum_{i=1}^{m_{j}} j \mu_{j, i}(k) \\
& \geqslant q+(p+1)^{k}-(p+1)-\sum_{l \mid k, l<k}\left[q+(p+1)^{l}-(p+1)\right]-\sum_{j \mid k} \sum_{i=1}^{m_{j}} j \mu_{j, i}(k) .
\end{aligned}
$$

下面证明

$$
\sum_{j \mid k} \sum_{i=1}^{m_{j}} j \mu_{j, i}(k) \leqslant k L,
$$

这里 $L$ 表示 $R$ 的所有吸引花瓣循环的数目. 事实上, 设 $\Gamma_{j, i}$ 是周期为 $j \mid k$ 的一条 周期轨道, 使得 $\mu_{j, i}(k)>0$, 则它是有理中性的, 从而存在最小的整数 $m \geqslant 1$, 使 
得 $\left[\left(R^{j}\right)^{\prime}\left(z_{j, i}\right)\right]^{m}=1$. 于是 $(j m) \mid k$ 并且由 (1) 式及其后的说明有

$$
R^{j m}(z)=z+c\left(z-z_{0}\right)^{\nu m+1}[1+o(1)],
$$

这里 $c \neq 0$ 为常数, $\nu$ 表示 $R$ 的围绕轨道 $\Gamma_{j, i}$ 的吸引花瓣循环的数目. 由 (30) 和 (2) 及 (23) 式 (取 $l=k$ ) 可知, $\mu_{j, i}(k)=\nu m$, 从而 $j \mu_{j, i}(k)=m j \nu \leqslant k \nu$. 由此即知 (29) 式成立.

再由引理 2 和 3 , 有

$$
L \leqslant 2 d-2=2 p, \quad m_{k} \leqslant 2 d-2=2 p .
$$

由 (28)、(29)、(31) 式及 $N_{p}(k)=k n_{k}$ 和 $N_{r p}(k)=k\left(n_{k}-m_{k}\right)$ 就有

$$
\begin{aligned}
& N_{p}(k) \geqslant(p+1)^{k}-\sum_{j \mid k, j<k}(p+1)^{j}-2 k p=d^{k}-\sum_{j \mid k, j<k} d^{j}-2 k(d-1), \\
& N_{r p}(k) \geqslant(p+1)^{k}-\sum_{j \mid k, j<k}(p+1)^{j}-4 k p=d^{k}-\sum_{j \mid k, j<k} d^{j}-4 k(d-1) .
\end{aligned}
$$

定理证毕.

定理 1 的证明 假设 $R$ 没有周期为 $k$ 的排斥周期点，则由定理 2 知

$$
\begin{aligned}
d^{k} & \leqslant 4 k(d-1)+\sum_{j \mid k, j<k} d^{j} \\
& \leqslant 4 k(d-1)+\frac{d}{d-1}\left(d^{[k / 2]}-1\right) \\
& \leqslant 4 k(d-1)+2\left(d^{[k / 2]}-1\right) \\
& \leqslant(4 k+2)\left(d^{k / 2}-1\right)<5 k d^{k / 2},
\end{aligned}
$$

这里 $[k / 2]$ 是不超过 $k / 2$ 的最大整数, 从而

$$
d<(5 k)^{2 / k} \text {. }
$$

令 $\phi(x)=(5 x)^{2 / x}$, 则当 $x>1$ 时, $\phi^{\prime}(x)=-2(5 x)^{2 / x}[\log (5 x)-1] / x^{2}<0$, 从而 $\phi(x)$ 单调递减. 于是由 (36) 式, 当 $k \geqslant 12$ 时, $d<60^{2 / 12}<2$, 这说明 $k \leqslant 11$. 再 由 (36) 式知, 当 $k \geqslant 7$ 时, $d<35^{2 / 7}<3$, 于是当 $7 \leqslant k \leqslant 11$ 时, $d=2$. 再置

$$
f_{k}(d)=d^{k}-\left(4 k(d-1)+2\left(d^{[k / 2]}-1\right)\right),
$$

则由 $(35)$ 式有 $f_{k}(d) \leqslant 0$. 然而我们有 $f_{11}(2)=1942, f_{10}(2)=922, f_{9}(2)=446$, $f_{8}(2)=194, f_{7}(2)=86$, 于是 $k \leqslant 6$. 但我们还有

$$
\begin{aligned}
f_{6}(d)= & (d-2)^{6}+12(d-2)^{5}+60(d-2)^{4} \\
& +158(d-2)^{3}+228(d-2)^{2}+144(d-2)+26 \\
f_{5}(d)= & (d-2)^{5}+10(d-2)^{4}+40(d-2)^{3}+78(d-2)^{2}+52(d-2)+6, \\
f_{4}(d)= & (d-3)^{4}+12(d-3)^{3}+52(d-3)^{2}+80(d-3)+33 \\
f_{3}(d)= & (d-4)^{3}+12(d-4)^{2}+34(d-4)+22 \\
f_{2}(d)= & (d-9)^{2}+8(d-9)+1
\end{aligned}
$$


于是由 $f_{k}(d) \leqslant 0$ 及 $d \geqslant 2$ 知 $k \leqslant 4$, 并且当 $k=4$ 时, $d=2$, 当 $k=3$ 时, $2 \leqslant d \leqslant 3$, 当 $k=2$ 时, $2 \leqslant d \leqslant 8$. 定理证毕.

\section{参考文献}

1 任福尧. 复解析动力系统. 上海: 复旦大学出版社, 1997

2 Carleson L, Gamelin T W. Complex Dynamics. New York, Berlin, Heidelberg: Springer, 1993

3 Baker I N. Fix-points of polynomials and rational functions. J London Math Soc, 1964, 39(2): 615-622

4 Bergweiler W. Periodische Punkte bei der Iteration ganzer Funktionen. Habilitationsschrift, RheinischWestfälische Techn. Hochsch: Aachen, 1991

5 Bergweiler W. Ahlfors theory and complex dynamics: periodic points of entire functions. In: Complex Dynamics and Related Fields. RIMS Kokyuroku, 2002, 1269: 1-11

6 Fatou P. Sur les équations fonctionelles. Bull Soc Math France, 1919, 47: 161-271

7 Julia G. Sur l'itération des fonctions rationelles. J Math Pures Appl, 1918, 4(7): 47-245

8 Bergweiler W. An introduction to complex dynamics. Textos Mat Sér B, 1995, 6: 1-37

9 Qiu L, Wu S J. Weakly repelling fixed points and multiply-connected wandering domains of meromorphic functions. Sci China Ser A, 2006, 49(2): 267-276

10 Essén M, Wu S J. Repulsive fixpoints of analytic functions with application to complex dynamics. J London Math Soc, 2000, 62(2): 139-148

11 Shishikura M. On the quasi-conformal surgery of rational functions. Ann SciÉcole Norm Sup, 1987, 20(4): 1-29

\section{附录：例 6 的计算}

本文中所有的例子都是借助计算软件 Maple 找到的. 这里就例 6 的验证计算过程作一个 说明. 首先用命令

$>\quad R:=z+\left((c-1) * z^{\wedge} 7+a\right) * z /\left(z^{\wedge} 7+2882600\right) ; \quad a:=-86017536 * c^{\wedge} 7+156417024 * c^{\wedge} 6-$ $67928160 * c^{\wedge} 5-56966440 * c^{\wedge} 4-17020121 * c^{\wedge} 3+3791327 * c^{\wedge} 2-24046371 * c-5852675$; 输入 $R(z)$ 和 $a$. 记住常数 $c$ 满足 $0<|c|<1$, 这是为了保证 $R$ 是一个 8 次有理函数及 $\infty$ 是 $R$ 的排斥不动点. 然后用命令

$>\quad Q 2:=\operatorname{numer}\left(\right.$ factor $\left.\left(-z+R+\left((c-1) * R^{\wedge} 7+a\right) * R /\left(R^{\wedge} 7+2882600\right)\right)\right) ;$

来计算 $Q_{2}(z)$, 这里 $Q_{2}(z)$ 表示 $R^{2}(z)-z$ 的分子, 是一个次数为 64 的多项式 (关于 $z$ ) (参见本文 引理 4), 但它不是首一的. 这个命令同时也对 $Q_{2}(z)$ 进行了因式分解, 它有因式 $\left((c-1) z^{7}+a\right) z$. 再用命令

$>\quad W:=\operatorname{sort}\left(\operatorname{collect}\left(Q 2 / z /\left((c-1) * z^{\wedge} 7+a\right), z\right), z\right) ;$

我们得到一个首项系数为 $c^{7}+c^{8}$ 的多项式 $W$, 并且对 $z$ 进行了合并和排序. 由此可看出 $W$ 是一个 56 次的多项式, 并且只含有 $z^{7 n}$ 项. 按照例 6 的要求, 我们希望 $W$ 是某个多项式 $H$ 的平方, 因此现在令

$>\quad H:=z^{\wedge} 28+a 1 * z^{\wedge} 21+a 2 * z^{\wedge} 14+a 3 * z^{\wedge} 7+a 4 ;$

这里 $a 1, a 2, a 3, a 4$ 是待定常数. 现在计算 $W-\left(c^{7}+c^{8}\right) H^{2}$,

$>\quad T:=\operatorname{sort}\left(\operatorname{collect}\left(\operatorname{normal}\left(H^{\wedge} 2-W /\left(c^{\wedge} 7+c^{\wedge} 8\right)\right.\right.\right.$, expanded), $\left.\left.z\right), z\right)$;

然后通过令 $T$ 的 $49,42,35,28$ 次项的系数为 0 , 先用 $c$ 将 $a 1, a 2, a 3, a 4$ 依次表示出来, 然后将 这些表达式代入, 再对 $T$ 进行因式分解

$>\quad T:=$ factor $(T)$; 
我们看到有一个因式为 $1536 c^{8}+1536 c^{7}+1120 c^{6}+840 c^{5}+721 c^{4}+308 c^{3}+126 c^{2}+60 c+25$, 从而当常数 $c$ 满足 $1536 c^{8}+1536 c^{7}+1120 c^{6}+840 c^{5}+721 c^{4}+308 c^{3}+126 c^{2}+60 c+25=0$ 时, 有 $W \equiv\left(c^{7}+c^{8}\right) H^{2}$. 最后用命令

$>$ fsolve $\left(1536 * c^{\wedge} 8+1536 * c^{\wedge} 7+1120 * c^{\wedge} 6+840 * c^{\wedge} 5+721 * c^{\wedge} 4+308 * c^{\wedge} 3+126 *\right.$ $c^{\wedge} 2+60 * c+25, c$, complex);

可知满足方程 $1536 c^{8}+1536 c^{7}+1120 c^{6}+840 c^{5}+721 c^{4}+308 c^{3}+126 c^{2}+60 c+25=0$ 的 $c$ 都在去心单位圆内. 这样就验证了例 6 . 